\title{
VERAPAMIL PLUS NITROGLYCERIN SOLUTION MAXIMALLY PRESERVES ENDOTHELIAL FUNCTION OF THE RADIAL ARTERY: COMPARISON WITH PAPAVERINE SOLUTION
}

Guo-Wei He, MD, PhD

A rterial grafts have been increasingly used for coronary artery bypass grafting (CABG) since the successful use of the internal thoracic artery

From the Division of Cardiothoracic Surgery, Department of Surgery, University of Hong Kong, Grantham Hospital, Hong Kong.

Supported by the Hong Kong Research Grants Council (grant 338/048/0004). Partially supported by Committee of Research and Conference Grant (337/048/0018, 335/048/0079) and University Grant (014/048/9602 and 344/048/0001), The University of Hong Kong.

Received for publication June 19, 1997; revisions requested July 30, 1997; revisions received Jan. 19, 1998; accepted for publication Jan. 21, 1998.

Address for reprints: Professor Guo-Wei He, MD, PhD, Chair of Cardiothoracic Surgery, University of Hong Kong, Grantham Hospital, 125 Wong Chuk Hang Rd., Aberdeen, Hong Kong.

Copyright (c) 1998 by Mosby, Inc.

$0022-5223 / 98 \$ 5.00+0 \quad \mathbf{1 2 / 1 / 8 9 0 7 9}$
(ITA) for grafting. ${ }^{1}$ Additional arterial grafts are the gastroepiploic artery, ${ }^{2}$ the inferior epigastric artery, ${ }^{3}$ and the radial artery. ${ }^{4-8}$ Occasionally, other arteries such as splenic or subscapular arteries have been reported as grafts. The ITA is probably the first choice as the arterial graft in most institutions. A definitive opinion with regard to the second choice of arterial grafts has not been formed. However, in some institutions, ${ }^{8}$ and recently at the University of Hong Kong, Grantham Hospital, the radial artery has become the preferred arterial graft, next to the ITA.

According to the functional classification devised by my colleague and myself, ${ }^{9}$ the radial artery belongs to the type III arterial graft-a type of artery that is more spastic than type I arteries (such as the ITA and inferior epigastric artery). From our experience in accordance with others, ${ }^{8}$ contraction (or spasm) of the radial artery is almost inevitably 
encountered during surgical dissection. The spastic characteristic of the radial artery warrants the use of vasodilators during harvesting. In fact, the use of vasodilators is a key step in the revival of the radial artery. ${ }^{8}$ In the standard protocol suggested by Acar and associates, ${ }^{8}$ diltiazem is used as the systemic vasodilator and papaverine is used topically during harvesting.

My colleague and $\mathrm{I}^{10}$ have recently provided data to use alternative vasodilators for the radial artery. We demonstrated that the combination of verapamil and nitroglycerin solution (VG solution), balanced to $\mathrm{pH}$ 7.4, may provide a rapid onset, a complete relaxation, and a long-lasting vasorelaxant effect when used to prepare the radial artery for grafting. ${ }^{10}$ To choose a calcium antagonist to be used for the radial artery graft, we reviewed our previous pharmacologic studies, which compared the effect of calcium antagonists from three chemically divergent groups-dihydropyridine (e.g., nifedipine), phenylalkylamines (e.g., verapamil), and benzothiazepines (e.g., diltiazem) in the human ITA $^{11}$ and other vascular tissues (canine coronary arteries $^{12}$ ). Consolidating the aforementioned information, we designed the University of Hong Kong (UHK) protocol for use of the radial artery as a graft for CABG (see Material and methods).

The purpose of this study was to examine the preservation of endothelial function of the radial artery by using the UHK protocol to harvest the radial artery for grafting.

\section{Material and methods \\ UHK protocol \\ Preoperative}

1. Allen test is used for both arms.

2. Doppler flow examination is used for the ulnar artery during the Allen test.

\section{Intraoperative}

1. Use VG solution topically during harvesting.

2. The radial artery is removed as soon as it is dissected from the arm and stored in the VG solution at room temperature.

3. Once the harvesting of the radial artery is started, low-dose verapamil $(0.5 \mathrm{mg} / \mathrm{hr} ; 5 \mathrm{mg}$ in $100 \mathrm{ml} 5 \%$ dextrose in water, intravenously, at the rate of 10 $\mathrm{ml} / \mathrm{hr}$ ) is given systematically.

\section{Postoperative}

1. Intravenous verapamil is given at the same dose until the patient is able to take oral verapamil.

2. Oral verapamil, $240 \mathrm{mg}$ per day, is given for at least 12 months, maybe longer; $120 \mathrm{mg}$ as the initial testing dose is recommended. If not tolerated by the patient, mainly because of bradycardia, the alternative is to give nifedipine (retard preparation), $20 \mathrm{mg}$ per day. No $\beta$-blockers are given after the operation to avoid severe bradycardia as it is seen in the combined use of verapamil and $\beta$-blockers.

Harvesting technique. The surgical dissection of the radial artery basically follows the technique described previously. 8,13 Once the harvesting of the radial artery is started, low-dose verapamil $(0.5 \mathrm{mg} / \mathrm{hr})$ is given intravenously. A curved skin incision parallel to the course of the radial artery is used. The radial artery is carefully dissected with its accompanying veins and the connective tissue. The side branches are carefully identified and clamped by small-sized hemoclips. During harvesting, VG solution may be used to bathe the radial artery. The artery is removed as soon as it is dissected out. Heparin is given before the artery is removed. The radial artery is immediately placed into a bowl containing enough volume of VG solution to be completely immersed. The gauze-wrap technique is not used, because it may not be as efficient as the immersion method to allow the artery (both the adventitia and the intima) to have complete contact with the solution. The radial artery is checked after at least 15 minutes' immersion in the VG solution, allowing full relaxation of the artery. ${ }^{10} \mathrm{~A}$ 24-gauge plastic arterial puncture needle sheath is inserted into the radial artery (usually the proximal end) and the artery is gently held with the fingers. No tie is necessary. Clean VG solution is injected through the needle to flush (not distend) the radial artery with the other end freely open. This gentle flush at low pressure will not distend the artery, and its purpose is to flush out any blood clots and to test the patency of the artery. This flushing procedure is not done specifically for detecting side-branch leakage, but some large branches may be detected at this time. For easy detection of leakage, one may use heparinized blood to flush the radial arterial lumen and then use VG solution to flush again. In our experience, there is no need to flush the radial arterial lumen with blood for this purpose. If the radial artery is to be used after vein grafts, it is placed back in the clean VG solution and stored in the bowl until use. The distal end of the radial artery is anastomosed to the coronary artery with an 8-0 or 7-0 Prolene running stitch (Ethicon, Inc., Somerville, N.J.). When the heart is resuscitated, usually there is back flow from the proximal end. Side branch bleeding may be easily detected during this period and stopped by a hemoclip. A $3.6 \mathrm{~mm}$ punch is used to make an oval hole in the ascending aorta. The proximal end is directly anastomosed to the aorta with a $7-0$ or $6-0$ Prolene stitch.

The components of the VG solution are as follows ${ }^{10}$ : verapamil hydrochloride $5 \mathrm{mg}$; nitroglycerin $2.5 \mathrm{mg}$; heparin 500 units; $8.4 \% \mathrm{NaHCO}_{3} 0.2 \mathrm{ml}$; Ringer's solution $300 \mathrm{ml}$. This solution gives a concentration of verapamil or nitroglycerin of about $30 \mu \mathrm{mmol} / \mathrm{L}$ in an isotonic solution with a $\mathrm{pH}$ of 7.4 .

Endothelial function test. The radial arterial segments were collected from patients undergoing $\mathrm{CABG}$ with this graft. Approval to use discarded radial artery tissue was 
given by the Ethics Committee of Grantham Hospital. After the arterial grafts were dissected, the required length was carefully measured. Any discarded segments of radial artery were immediately collected and placed into a container with oxygenated physiologic (Krebs) solution maintained at $4^{\circ} \mathrm{C}$ and then transferred to the laboratory. The vessels were placed in a glass dish and dissected out from their surrounding connective tissue. The arteries were cut into $3 \mathrm{~mm}$ long rings and then suspended on wires in organ baths. ${ }^{12}$ The number of the rings provided by each patient varied from two to six. The Krebs solution had the following composition (in millimoles per liter): $\mathrm{Na}^{+} 144, \mathrm{~K}^{+} 5.9, \mathrm{Ca}^{2+} 2.5, \mathrm{Mg}^{2+} 1.2, \mathrm{Cl}^{-} 128.7, \mathrm{HCO}_{3}{ }^{-} 25$, $\mathrm{SO}_{4}^{-2} 1.2, \mathrm{H}_{2} \mathrm{PO}_{4}^{-} 1.2$, and glucose 11 . The solution was aerated with a gas mixture of $95 \%$ oxygen and $5 \%$ carbon dioxide at $37^{\circ} \pm 0.1^{\circ} \mathrm{C}$.

Organ-bath technique. A normalization technique was used to set the vascular rings at a pressure comparable with the in vivo pressure. The details of the technique were published before. ${ }^{12,15}$

The endothelium was intentionally preserved by cautiously dissecting and mounting the rings. My colleagues and I previously found that this technique allowed the experiments to be carried out with an intact endothelium, as determined by the functional relaxation response to acetylcholine in the canine ITA $^{12}$ and to substance $\mathrm{P}$ and A23187 in the porcine ${ }^{16}$ coronary artery. We have also shown that the endothelium was functionally preserved in the isolated human ITA $^{17-19}$ and radial artery. ${ }^{20}$

Protocol. The rings were randomly distributed to different experimental groups. In each group, the rings were taken from at least four patients.

Endothelial function in control radial artery. Segments were taken from the radial artery that was not treated with VG solution. After the normalization procedure, the vascular rings were equilibrated for at least 1 hour. The endothelial function was indexed by endothelium-dependent relaxation. The relaxation was expressed as percentage relaxation of the precontraction induced by U46619 (10 nmol/L), a stable thromboxane $A_{2}$ mimetic, which has been demonstrated to be a strong vasoconstrictor in the human radial artery. ${ }^{20}$ Both receptor-mediated and nonreceptor-mediated endothelium-dependent relaxation were tested. Only one concentration-relaxation curve was established in each radial artery ring.

RECEPTOR-MEDIATED ENDOTHELIUM-DEPENDENT RELAXATION. This relaxation was induced by acetylcholine, a receptor-mediated endothelium-derived relaxing factor (EDRF) agonist.

NON-RECEPTOR-MEDIATED ENDOTHELIUM-DEPENDENT RELAXATION. This relaxation was induced by calcium ionophore A23187 (calcimycin), a non-receptor-mediated EDRF stimulus.

Endothelial function in radial artery treated with VG solution. Before the anastomosis, to ensure adequate length, the radial artery graft was again carefully measured. The redundant part of the radial artery was cut off and collected for the experiments. By this time, the sample of the radial artery usually had been immersed in VG solution for 30 to 45 minutes. Endothelial function was tested in those arteries according to the protocol in the control arteries, as described earlier.
In some radial artery rings, the endothelium was removed mechanically by using a fine wood stick moistened with Krebs solution to gently rub the intima of the rings. ${ }^{17}$ The relaxation to substance P or A23187 was tested in those rings to demonstrate the endothelium dependence of the relaxation.

Comparison between $V G$ and papaverine solutions in preservation of endothelial function in the radial artery. Radial artery segments were immersed either in VG solution as mentioned earlier or in papaverine solution (60 $\mathrm{mg}$ in $60 \mathrm{ml}$ normal saline solution as used clinically) at room temperature for 45 minutes. The $\mathrm{pH}$ of the papaverine solution measured in this study was 5.75 . The arteries were then cut into $3 \mathrm{~mm}$ segments and mounted in the organ bath. Acetylcholine- and A23187-mediated relaxation was compared between the rings treated with VG and those treated with papaverine solution.

Data analysis. The relaxation of the radial artery to VG solution was expressed as percentage of precontraction (to U46619 $10 \mathrm{nmol} / \mathrm{L}$ ). $\mathrm{EC}_{50}$ was calculated from the logistic curve-fitting technique. ${ }^{12,16}$

Unpaired Student's $t$ test was used to compare the contraction force or the percentage relaxation between two groups.

Drugs. Drugs used in this study and their sources were as follows: verapamil (Knoll AG, Germany), nitroglycerin (G. POHL-BOSKAMP, GmbH \& Co., Germany). Other chemicals were purchased from Sigma Chemical Company (St. Louis, Mo.).

\section{Results}

Sixty-seven radial artery rings were studied. The diameter at a pressure of $100 \mathrm{~mm} \mathrm{Hg}$ (D100) was $2.7 \pm 0.6 \mathrm{~mm}$. The transmural pressure at 0.9 $\mathrm{D} 100^{12,15}$ was $66.5 \pm 1.8 \mathrm{~mm} \mathrm{Hg}$. The resting force was $2.3 \pm 0.3 \mathrm{gm}$.

In acetylcholine experiments, U46619 induced $12.8 \pm 2.3 \mathrm{gm}$ force in the control arteries versus $14.2 \pm 0.9 \mathrm{gm}(p=0.6,95 \%$ confidence interval for the difference of the mean [95\% CI]: 1.6, $35.8 \mathrm{gm}$ ) in the radial artery segments treated with the VG solution. In the A23187 experiments, U46619 induced $8.5 \pm 0.5$ versus $8.8 \pm 0.5$ gm force $(p=0.7$, $95 \%$ CI: $0.3,5.0 \mathrm{gm})$. U46619 induced $7.2 \pm 2.1 \mathrm{gm}$ contraction in papaverine-treated segments versus $8.9 \pm 0.7 \mathrm{gm}$ in VG-treated radial artery segments ( $p=0.3,95 \%$ CI: $0.07,1.1 \mathrm{gm}$ ) for acetylcholine experiments and $5.9 \pm 1.5 \mathrm{gm}$ contraction in papaverine-treated segments versus $8.8 \pm 0.5 \mathrm{gm}$ in VG-treated radial artery segments $(p=0.2,95 \% \mathrm{CI}$ : $0.02,0.3 \mathrm{gm})$.

Receptor-mediated endothelium-dependent relaxation by acetylcholine (Fig. 1, $a$ ). Acetylcholine induced $27.5 \% \pm 5.0 \%$ relaxation $(n=7)$ in the control radial artery versus $23.9 \% \pm 3.9 \%$ relaxation $(n=6)$ in the radial artery segments treated 


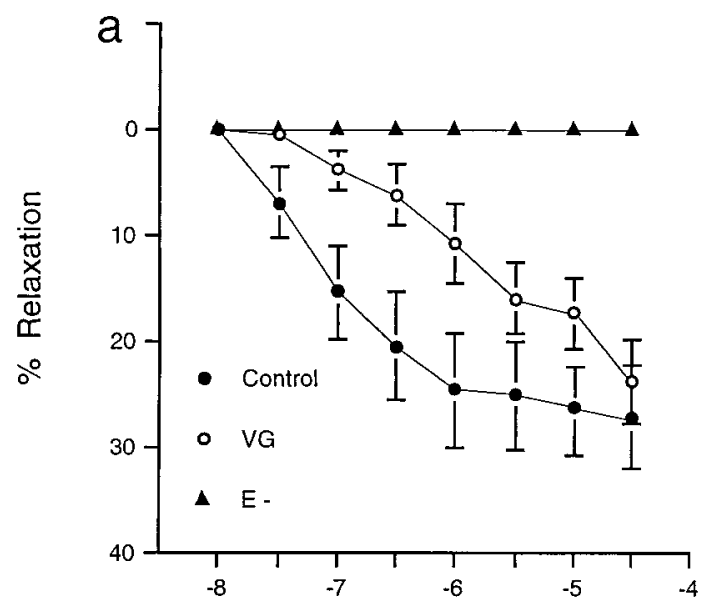

Acetylcholine $\log M$

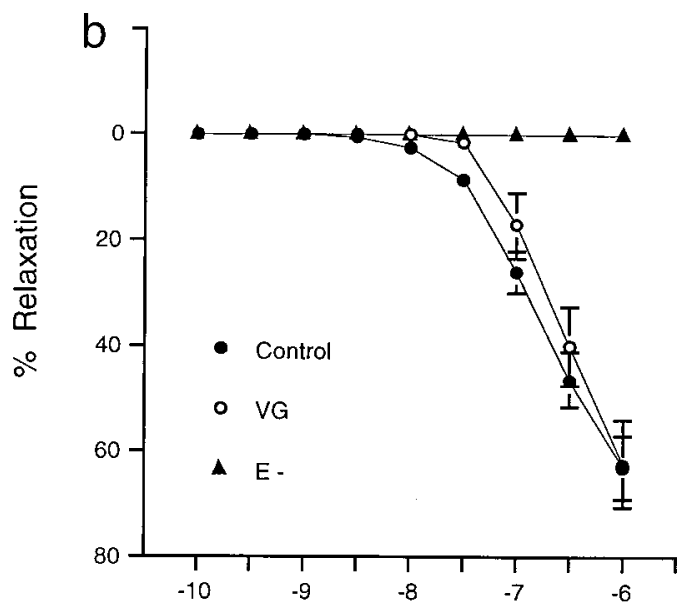

A23187 $\log M$

Fig. 1. Average concentration-relaxation curve to acetylcholine (a) and calcium ionophore (A23187, b) in the human radial artery segments precontracted with U46619 (10 nmol/L). Symbols represent average data from a number of vessels. a, Control, $n=7 ; V G$, radial artery segments treated with VG (verapamil plus nitroglycerin solution, $n=6) ; E$-, endothelium-denuded radial artery segments $(n=4)$. b, Control, $n=13$; $V G$, radial artery segments treated with VG (verapamil plus nitroglycerin solution, $n=6$ ); $E$-, endothelium-denuded radial artery segments $(n=4)$.

with VG solution ( $p=0.6$, unpaired $t$ test, 95\% CI: $0.4 \%, 8.4 \%$ ).

However, the VG treatment increased the $\mathrm{EC}_{50}$ $(-5.88 \pm 0.22$ vs $-6.97 \pm 0.14 \log \mathrm{mol} / \mathrm{L}, p=0.001$, 95\% CI: $0.09,1.97 \log \mathrm{mol} / \mathrm{L})$.

Non-receptor-mediated endothelium-dependent relaxation by $\mathbf{A 2 3 1 8 7}$ (Fig. 1, b). A23187 induced $62.9 \% \pm 6.0 \%$ relaxation of the U46619-induced precontraction $(n=13)$. There was no significant difference between this group and the VG-treated radial artery segments $(62.3 \% \pm 8.4 \%, n=6, p=$ 0.96; unpaired $t$ test; $95 \%$ CI: $0.2 \%, 3.5 \%$ ).

The $\mathrm{EC}_{50}$ was $-6.68 \pm 0.1$ in the control rings versus $-6.66 \pm 0.05 \log \mathrm{mol} / \mathrm{L}$ in the VG-treated rings ( $p=0.9$, unpaired $t$ test; 95\% CI: 1.61, $31.1 \log$ $\mathrm{mol} / \mathrm{L})$.

In endothelium-denuded radial artery rings, neither acetylcholine nor A23187 $(n=4)$ induced any relaxation in the radial artery, and this confirmed that the acetylcholine- and A23187-induced relaxation in the present study was endothelium-dependent (Fig. 1, $a$ and $b$ ).

Comparison between VG and papaverine solutions. In the papaverine-treated radial artery, acetylcholine-induced relaxation was almost abolished $(3.3 \% \pm 2.6 \%$ compared with $23.9 \% \pm 3.9 \%$ with VG treatment, $p<0.001$, unpaired $t$ test; 95\% CI:
$0.5 \%, 9.8 \%$, Fig. $2, a$ ). In addition, A23187 induced significantly less relaxation $(39.7 \% \pm 5.2 \%)$ than that $(62.3 \% \pm 8.4 \%)$ in the VG-treated radial artery $(p=0.02$, unpaired $t$ test; 95\% CI: $0.4 \%, 7.4 \%$ ) (Fig. $2, b)$. Because the acetylcholine-induced relaxation was abolished in some rings after treatment with papaverine, the $\mathrm{EC}_{50}$ value could not be calculated in these arteries. However, there was no difference with regard to the $\mathrm{EC}_{50}$ in $\mathrm{A} 23187$ experiments $(-6.69 \pm 0.09$ vs $-6.66 \pm 0.05 \log \mathrm{mol} / \mathrm{L}, p=0.8$, unpaired $t$ test; 95\% CI: $0.05,0.98 \log \mathrm{mol} / \mathrm{L})$.

\section{Discussion}

Operative results (early mortality and long-term patency) of CABG at large depend on the function of the graft. For the radial artery, the major concern is its spastic characteristic, which led to the early abandonment of this arterial graft. As to the longterm patency, apart from the necessary spasmolytic treatment, preservation of endothelium is vitally important. Vascular endothelium derives a number of EDRFs that play an important role in vasorelaxation and in inhibition of platelet aggregation. When endothelium is impaired, the antiplatelet function of EDRFs (such as nitric oxide and prostaglandin $\mathrm{I}_{2}$ ) is lost and platelets attach to the area denuded of endothelium. The coagulation cascade is 


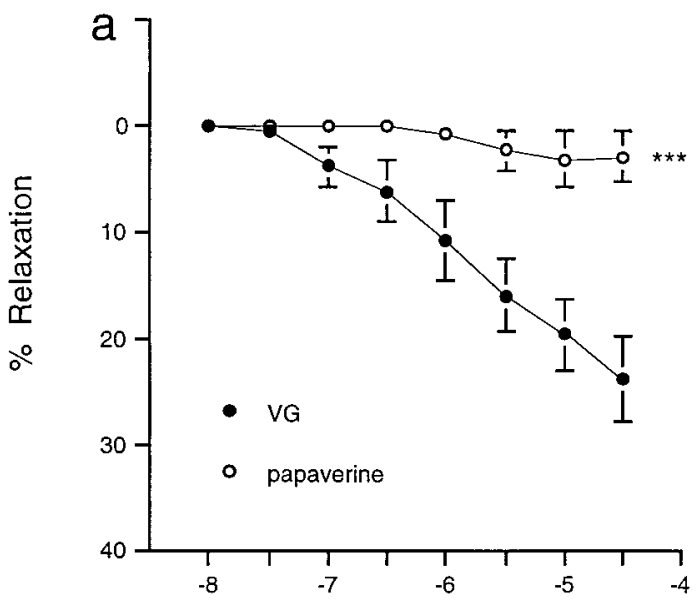

Acetylcholine $\log M$

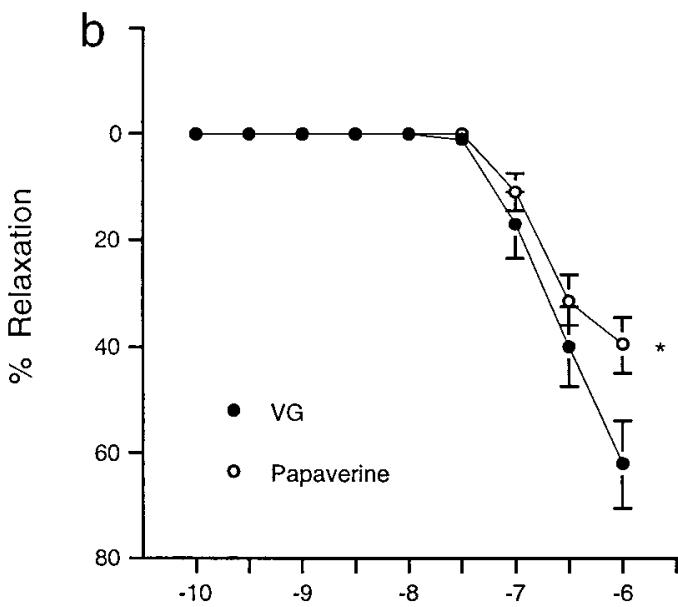

A23187 $\log M$

Fig. 2. Average concentration-relaxation curve to acetylcholine (a) and calcium ionophore (A23187) (b) in the human radial artery segments precontracted with U46619 (10 nmol/L). Symbols represent average data from a number of vessels. $V G$, Radial artery segments treated with VG (verapamil plus nitroglycerin solution); Papaverine, radial artery segments treated with papaverine solution $(60 \mathrm{mg} / 60 \mathrm{ml}$ saline solution, $n=10)$. ${ }^{*} p<0.05$ and ${ }^{* * *} p<0.001$ at the maximal relaxation compared with VG. a, $n=6$ for VG and $n=7$ for papaverine; $\mathbf{b}, n=6$ for $\mathrm{VG}$ and $n=10$ for papaverine.

activated by aggregating platelets and by thrombin. Thrombus then forms. This becomes the basis for later growth and development of atherosclerotic plaque and may lead to graft occlusion. Therefore an ideal method to harvest the radial artery (1) should be antispastic and (2) should allow maximum preservation of endothelial function. These principles prompted my colleagues and me to search for a method fulfilling these criteria in the harvesting of the radial artery.

The reported methods (Table I) to harvest the radial artery include (1) systemic use of diltiazem once the harvesting is started and (2) use of papaverine alone ${ }^{14}$ or mixed with blood" ${ }^{8,13}$ for "gentle hydrostatic dilation" of the artery to achieve antispastic effect and to check leaking. However, if a graft is fully relaxed, there is no need to mechanically dilate it because mechanical distention (dilation) may impair the endothelium or smooth muscle, particularly when the distention pressure is high. In fact, the distention pressure is difficult to control during harvesting.

In a previous study, my colleague and I have reported the advantages of using VG solution over papaverine in the radial artery. ${ }^{10}$ These involve (1) more rapid onset and (2) neutral $\mathrm{pH}$. Our experi- ments have shown a more rapid relaxation induced by the VG solution than papaverine within the first 10 minutes. Perhaps more importantly, VG solution is neutral $(\mathrm{pH}=7.4)$ whereas papaverine is acidic, as measured in this study $(\mathrm{pH}=5.75$ at the clinically used concentration in the saline solution). It has been reported ${ }^{21}$ that a white precipitate sometimes forms when papaverine is added to electrolyte solution (Plasma-Lyte; pH approximately 7.4) and papaverine is relatively unstable in nonacidic solution. Acidic solution has been shown to damage the endothelium. ${ }^{22}$ This is more important in the preparation of the radial artery than of the ITA because the ITA is usually used as a pedicle and most surgeons only spray the vasodilator solution on the surface of the pedicle. Therefore the vasodilator does not directly contact the endothelium of the ITA unless the intraluminal injection is used. However, the radial artery is used as a free graft. No matter how the radial artery is immersed in the solution, whether as I do it or wrapped in a gauze with vasodilator solution, it is inevitable that the endothelium makes contact with the solution.

In general, the advantages of calcium antagonists are (1) high potency to inhibit the voltage-operated calcium channel (L-type calcium channel), which is 
Table I. Existing techniques to overcome spasm of the radial artery

\begin{tabular}{llr}
\hline \multicolumn{1}{c}{ Authors } & \multicolumn{1}{c}{ Topical } & Systemic \\
\hline Acar et al $^{8}$ & $\begin{array}{l}\text { Papaverine + blood } \\
\text { Dietle and Benoit }\end{array}$ & Diltiazem \\
& $\begin{array}{l}\text { Papaverine } \\
\text { Diltiazem } \\
\text { "if spasm is noted" }\end{array}$ & Diltiazem \\
Reyes, Frame, & $\begin{array}{c}\text { Papaverine } \\
\text { Brodman }\end{array}$ & Diltiazem \\
He & $60 \mathrm{mg}+60 \mathrm{ml}$ blood & Verapamil \\
& VG & \\
\hline
\end{tabular}

the primary mechanism for regulation of intracellular calcium concentration of vascular smooth muscle, ${ }^{23}$ and (2) long duration.

The effects of three chemically different calcium antagonists $^{24}$ have been compared in the coronary artery and CABGs. ${ }^{11,12}$ Among the three calcium antagonists, diltiazem is the least potent. For example, nifedipine is 15 -fold more potent than diltiazem with regard to the vasorelaxant effect in the most commonly used human arterial CABG, the ITA, as demonstrated in a previous study. ${ }^{11}$ However, nifedipine is not available for intravenous or topical use. Verapamil is more potent than diltiazem in the canine ITA $\left(\mathrm{EC}_{50}-5.73\right.$ vs $\left.-5.38 \log \mathrm{mol} / \mathrm{L}\right)$ and saphenous vein $(-6.74 \mathrm{vs}-6.30 \mathrm{log} \mathrm{mol} / \mathrm{L}) .{ }^{12}$ It has also been found that in the human saphenous vein diltiazem is significantly less potent than verapamil $\left(\mathrm{EC}_{50}-6.62 \mathrm{vs}-6.96 \log \mathrm{mol} / \mathrm{L}\right){ }^{25}$ Therefore we studied the effect of verapamil and nitroglycerin in the radial artery. ${ }^{10}$

Apart from the aforementioned, other advantages of verapamil have been reported. It has been suggested to have antiplatelet and antithrombotic effect in patients with coronary artery disease, ${ }^{26}$ to improve the prognosis in patients who have had an infarction, ${ }^{27}$ and to inhibit smooth muscle cell proliferation in experimental vein bypass grafts. ${ }^{28}$

The common disadvantages of calcium antagonists, on the other hand, are (1) that high vasoconstrictor selectivity may limit their effect under some circumstances when a vessel is contracted through receptor mechanisms and (2) that onset of action is slower than that with nitroglycerin, although the onset is more rapid than with papaverine. ${ }^{10}$ As demonstrated in the human ITA and radial artery, ${ }^{10}$ a combination of calcium antagonists with nitroglycerin may combine the advantages of both.

The major findings from the present study are that, in addition to the full relaxant effect in the radial artery found previously, the endothelial func- tion is maximally preserved after being immersed in the VG solution for 45 minutes. In my experiments, two mechanisms of endothelium-dependent relaxation were tested-receptor-mediated relaxation (by acetylcholine) and non-receptor-mediated relaxation (by the calcium ionophore A23187). In both experiments, the maximal relaxation mediated by EDRFs was not significantly different from that of the control radial artery. These results provide evidence that the VG solution does not impair the endothelial function after full exposure (immersion) during surgery. This is probably due to the neutral $\mathrm{pH}$ and the isotonic nature of this solution. In addition, nitroglycerin is an exogenous nitric oxide donor. It releases nitric oxide when it diffuses into the vascular smooth muscle, ${ }^{29}$ which is identical to endothelium-derived nitric oxide-one of the three major EDRFs. ${ }^{30}$ Therefore nitroglycerin would enhance but not impair endothelium-derived nitric oxide-mediated endothelial function.

In contrast, the present study demonstrates that after papaverine treatment, endothelial function in the radial artery is impaired (Fig. 2). This is most likely due to the acidic characteristic of papaverine solution $(\mathrm{pH}=5.75)$, as mentioned earlier. My colleague and $\mathrm{I}^{10}$ have previously demonstrated that VG solution has faster onset to relaxation of the radial artery than papaverine. In this study, I have demonstrated the superiority of VG to papaverine solution in the protection of endothelial function of the radial artery.

We have previously reported the excellent relaxation effect of VG solution in human saphenous vein $^{25}$ and ITA. ${ }^{10}$ In our practice, we use only one solution to prepare all CABGs, and this is extremely convenient for the surgical team.

I realize that results from in vitro experiments can only be carefully transferred to the clinical setting. The influence of the UHK protocol on long-term patency is still unknown. Angiographic studies are necessary to prove its efficacy. However, we have now used the UHK protocol in more than 75 patients receiving radial artery grafts. By using this protocol, we have always seen a "fully relaxed" radial artery before performing the anastomosis, and we have not encountered spasm of the radial artery during the operation.

In conclusion, the present study suggests that use of VG solution to prepare the radial artery for CABG provides a method that may maximally preserve the endothelial function of the radial artery. In contrast, papaverine solution may impair this func- 
tion. Together with the previous findings that VG solution fully relaxes the radial artery and overcomes spasm without distention or "hydrostatic dilation," this solution may be effectively and safely used to prepare the radial artery for CABG.

I gratefully acknowledge the excellent experimental work of Dr. Cheng-Qin Yang at the Cardiovascular Research Laboratory, Department of Surgery, University of Hong Kong, Grantham Hospital. The cooperation of the cardiologists, the technical assistance of the surgical medical officers at the Division of Cardiothoracic Surgery, and the Operating Theater nurses and technicians at Grantham Hospital are also gratefully acknowledged.

\section{REFERENCES}

1. Loop FD, Lytle BW, Cosgrove DM, et al. Influence of the internal-mammary-artery graft on 10-year survival and other cardiac events. N Engl J Med 1986;314:1-6.

2. Pym J, Brown PM, Charrette EJP, Parker JO, West RO. Gastroepiploic-coronary anastomosis: a viable alternative bypass graft. J Thorac Cardiovasc Surg 1987;94:256-9.

3. Puig LB, Ciongolli W, Cividanes GVL, et al. Inferior epigastric artery as a free graft for myocardial revascularization. J Thorac Cardiovasc Surg 1990;99:251-5.

4. Carpentier A, Guermonprez JZ, Deloche A, Frechette C, Dubost $\mathrm{C}$. The aorto-to-coronary radial artery bypass graft: a technique avoiding pathological changes in grafts. Ann Thorac Surg 1973;16:111-21.

5. Fisk RL, Bruoks CH, Callaghan JC, Dvorkin J. Experience with the radial artery graft for coronary bypass. Ann Thorac Surg 1976;21:513-8.

6. Carpentier A. Discussion of Geha AS, Krone RJ, McCormick JR, Baue AE. Selection of coronary bypass: anatomic, physiological, and angiographic considerations of vein and mammary artery grafts. J Thorac Cardiovasc Surg 1975;70: 414-31.

7. Chiu C-j. Why do radial artery grafts for aortocoronary bypass fail? A reappraisal. Ann Thorac Surg 1976;22:520-3.

8. Acar C, Jebara VA, Portoghese M, et al. Revival of the radial artery for coronary bypass grafting. Ann Thorac Surg 1992; 54:652-60

9. He G-W, Yang C-Q. Comparison among arterial grafts and coronary artery: an attempt at functional classification. J Thorac Cardiovasc Surg 1995;109:707-15.

10. He G-W, Yang C-Q. Use of verapamil and nitroglycerin solution for preparation of radial artery for coronary bypass grafting. Ann Thorac Surg 1996;61:610-4.

11. He G-W, Buxton B, Rosenfeldt F, Angus JA. Reactivity of human isolated internal mammary artery to constrictor and dilator agents: implications for treatment of internal mammary artery spasm. Circulation 1989;80(Suppl):I141-50.

12. He G-W, Angus JA, Rosenfeldt FL. Reactivity of the canine isolated internal mammary artery, saphenous vein, and coronary artery to constrictor and dilator substances: relevance to coronary bypass graft surgery. J Cardiovasc Pharmacol 1988;12:12-22.

13. Reyes AT, Frame R, Brodman RF. Technique for harvesting the radial artery as a coronary artery bypass graft. Ann Thorac Surg 1995;59:118-26.

14. Dietle CA, Benoit CH. Radial artery graft for coronary revascularization: technical considerations. Ann Thorac Surg 1995;60:102-10

15. He G-W, Buxton B, Rosenfeldt F, Wilson AC, Angus JA. Weak $\beta$-adrenoceptor-mediated relaxation in the human internal mammary artery. J Thorac Cardiovasc Surg 1989;97: 259-66.

16. He G-W, Yang C-Q, Graier WF, Yang J-A. Hyperkalemia alters EDHF-mediated hyperpolarization and relaxation in coronary arteries. Am J Physiol 1996;271:H760-7.

17. He G-W, Yang C-Q. Effects of thromboxane $\mathrm{A}_{2}$ antagonist GR32191B on prostanoid and nonprostanoid receptors in the human internal mammary artery. J Cardiovasc Pharmacol 1995;26:13-9.

18. He G-W, Yang C-Q, Mack MJ, Acuff TE, Ryan WH, Starr A. Interaction between endothelin and vasodilators in the human internal mammary artery. Br J Clin Pharmacol 1994;38: $505-12$.

19. He G-W, Shaw J, Hughes CF, et al. Predominant $\alpha_{1^{-}}$ adrenoceptor mediated contraction in the human internal mammary artery. J Cardiovasc Pharmacol 1993;21:256-63.

20. He G-W, Yang C-Q. Radial artery has higher receptorselective contractility but similar endothelium function compared to mammary artery. Ann Thorac Surg 1997;63:1346-52.

21. Cunningham JN. Papaverine hydrochloride preservation of vein grafts. J Thorac Cardiovasc Surg 1982;84:933-4.

22. Constantinides P, Rohmson M. Ultrastructural injury of arterial endothelium. 1. Effects of $\mathrm{pH}$, osmolarity, anoxia and temperature. Arch Pathol 1969;88:99-105.

23. Slish DF, Schultz D, Schwartz A. Molecular biology of calcium antagonist receptor. Hypertension 1992;19:19-24.

24. Borchard U. Calcium antagonists in comparison: view of the pharmacologist. J Cardiovasc Pharmacol 1994;24(suppl 2): s85-91.

25. He G-W, Rosenfeldt FL, Angus JA. Pharmacological relaxation of the saphenous vein during harvesting for coronary artery bypass grafting. Ann Thorac Surg 1993;55:1210-7.

26. Lacoste L, Lam JYT, Hung J, Waters D. Oral verapamil inhibits platelet thrombus formation in humans. Circulation 1994;89:630-4.

27. The Danish Study Group on Verapamil in Myocardial Infarction. Effect of verapamil on mortality and major events after acute myocardial infarction (The Danish Verapamil Infarction Trial II-DAVIT II). Am J Cardiol 1990;66:779-85.

28. Brauner R, Laks H, Drinkwater DC, et al. Controlled periadventitial administration of verapamil inhibits neointimal smooth muscle cell proliferation and ameliorates vasomotor abnormalities in experimental vein bypass grafts. J Thorac Cardiovasc Surg 1997;114:53-63.

29. Bassenge E. Coronary arterial dilators and venodilators. In: Singh BN, et al, editors. Cardiovascular pharmacology and therapeutics. New York: Churchill Livingstone; 1994. p. 164.

30. He G-W, Yang C-Q, Yang J-A. Depolarizing cardiac arrest and EDHF-mediated hyperpolarization and relaxation in coronary arteries: the effect and mechanism. J Thorac Cardiovasc Surg 1997;113:932-41. 